\title{
Parathyroid hormone or fibroblast growth factor 23? Which one is the main determinant of the hypophosphatemia after kidney transplantation?
}

\author{
Seyed Sadroddin Rasi Hashemi ${ }^{\mathbb{1}}$, Zahra Navarbaf ${ }^{*}{ }^{*}$, Amir Ghorbanihaghjo $^{2}$, Morteza Ghojazadeh $^{3}$, \\ Jalal Etemadi ${ }^{1}$, Sima Abediazar ${ }^{1}$
}

${ }^{1}$ Kidney Research Center, Tabriz University of Medical Sciences, Tabriz, Iran

${ }^{2}$ Biotechnology Research Center, Tabriz University of Medical Sciences, Tabriz, Iran

${ }^{3}$ Research Center of Evidence Based Medicine, Tabriz University of Medical Sciences, Tabriz, Iran

\begin{tabular}{l} 
A R T I C L E I N F O \\
\hline $\begin{array}{l}\text { Article Type: } \\
\text { Original }\end{array}$ \\
\hline Article History: \\
Received: 13 July 2018 \\
Accepted: 2 October 2018 \\
Published online: 30 October \\
\hline Keywords: \\
Fibroblast growth factor 23 \\
Parathyroid hormone \\
Hypophosphatemia \\
Kidney transplantation \\
Mineral and bone disorder \\
Chronic kidney disease \\
Glomerular filtration rate
\end{tabular}

\begin{abstract}
A B S T R A C T
Introduction: Kidney transplantation restores many of the disorders accompanying endstage renal disease (ESRD). However, hypophosphatemia is common complication after renal transplantation. High levels of fibroblast growth factor 23 (FGF23) and parathyroid hormone $(\mathrm{PTH})$ are two suspected factors determining the hypophosphatemia after kidney transplantation.

Objectives: This observational prospective study was carried out to clarify the role of mentioned factors in hypophosphatemia after kidney transplantation.

Patients and Methods: Living donor kidney transplant recipients which admitted to the ward of the renal transplantation, enrolled to the study. Parameters of bone and mineral metabolism including FGF23 and intact PTH levels were assessed.

Results: High FGF23 level before transplantation was related to lower phosphate levels at 3rd month after transplantation. PTH levels showed no relationship with hypophosphatemia after kidney transplantation.

Conclusion: High levels of FGF23 in ESRD patients undergoing kidney transplantation is an important determinant of hypophosphatemia in long-term follow up.
\end{abstract}

Implication for health policy/practice/research/medical education:

Recent researches pointed to persistent post-transplant elevations of FGF23 as having a major role in post-transplant hypophosphatemia.

Please cite this paper as: Rasi Hashemi S, Navarbaf Z, Ghorbanihaghjo A, Ghojazadeh M, Etemadi J, Abediazar S. Parathyroid hormone or fibroblast growth factor 23 ? Which one is the main determinant of the hypophosphatemia after kidney transplantation? J Renal Inj Prev. 2019;8(2):86-90. DOI: 10.15171/jrip.2019.17.

\section{Introduction}

Patients with chronic kidney disease (CKD) have abnormalities in maintaining the balance between intra- and extra-cellular ions such as calcium and phosphorus and the hormones that regulate this balance. Despite successful therapy of CKD patients by kidney transplant, CKD-mineral and bone disorder (CKD$\mathrm{MBD}$ ) continues to make problems in management of patients. Hypophosphatemia is frequently seen in posttransplantation period because of urinary phosphate wasting with restoration of renal clearance. Despite the recovery in most of the cases after a few weeks, hypophosphatemia persists in $6 \%$ to $27 \%$ of transplanted patients to months or years and cause complications such as muscle weakness, osteomalacia and metabolic encephalopathy (1-11).

Persistent elevation of parathyroid hormone (PTH) after transplantation was once considered as the main cause of hypophosphatemia, but recent researches also point to persistent post-transplant elevations of fibroblast growth factor 23 (FGF23) as playing a major role in post-transplant hypophosphatemia. In the kidney, FGF23 inhibits renal tubular phosphate reabsorption and promotes phosphate clearance. Fibroblast growth factor-23 also reduces levels 
of 1, 25-dihydroxyvitamin $\mathrm{D}$ and reduces phosphate absorption in intestine. Furthermore, FGF23 inhibits the normal action of parathyroid glands (12-20).

Fibroblast growth factor-23 level start to rise as glomerular filtration rate (GFR) declines below the normal level and continue to rise progressively as $\mathrm{CKD}$ progress and reaches to levels 100 to 1000 folds above normal in patients with end-stage renal disease (ESRD). High levels of FGF23 in ESRD thought to be the cause of continued elevation of FGF23 after successful kidney transplantation despite normal levels of phosphate $(1,2,10,11,15,21-25)$.

Although FGF23 levels are reported to decline rapidly after transplantation, there is limited information regarding its relationship to mineral metabolism in kidney transplant recipients in long-term follow up. As hypophosphatemia persists for a longer period after renal transplantation, the standard therapies such as phosphate supplement and calcitriol may not be effective because these agents can activate more FGF23 production. Calcimimetics and monoclonal antibodies against FGF23 are future hopes to reduce FGF23 levels and to diminish the chance of hypophosphatemia after transplant (2).

\section{Objectives}

This study aimed to assess the difference between FGF23 and PTH levels before and after transplantation and their relationship with hypophosphatemia after transplantation in a 6 month follow up of kidney recipients.

\section{Patients and Methods}

\section{Study design and population}

This study is prospective analytical-correlation study of living donor kidney transplant recipients admitted to the ward of transplantation of Emam Reza center, Tabriz University of Medical Sciences, Tabriz, Iran (between September 2015 and August 2016). Eligible patients were aged 15 to 75 with no systemic illness such as active malignancy, acquired immunodeficiency syndrome (AIDS), decompensated liver or respiratory disease and pregnancy. Fifty patients were enrolled in the study while 28 patients were followed until the end of the sixth month after kidney transplantation. Six patients were excluded at the first step because of comorbidities that made the transplantation impossible, three patients were unwilling to continue the study and were excluded during 6 months follow up, five patients died during 6 months follow up, four patients suffered from hyper-acute transplant rejection and undergone nephrectomy in the first step after transplantation. Additionally transplanted kidney function impairment happened for four patients during 6 months follow up. Therefore, 22 patients out of 50 patients were excluded from the study during 6 months follow up.

\section{Procedures, assays and calculations}

Serum samples were collected before transplantation, then 1 week, 3 months and 6 months after transplantation recollected and stored in $-70^{\circ} \mathrm{C}$. Serum FGF23 levels were determined by enzyme-linked immunosorbent assay (ELISA) method (Wuhan Eiaab Science CO., Wuhan, China). Serum 25 hydroxyvitamin D levels were determined by ELISA; (DIAsource ImmunoAssays S.A. Belgium) and serum intact PTH (iPTH) levels by ELISA; (Biomerica, INC. Irvine, USA). Serum calcium and phosphorus levels were measured using standard methods. Reference levels of 2.6-4.5 mg/dL and 8.6- 10.3 $\mathrm{mg} / \mathrm{dL}$ were used for serum phosphorus and calcium levels respectively. Serum urea and creatinine levels were measured in each step to control graft function and exclude those with impaired renal function. All participants get the same protocol of immunosuppression including glucocorticoids, calcineurin inhibitors and antimetabolites after transplant.

\section{Ethical issues}

The research followed the tenets of the Declaration of Helsinki. The Ethics Committee of Tabriz University of Medical Sciences approved this study. Written informed consent was obtained from all participants. All procedures performed in studies involving human participants were in accordance with the ethical standards of the ethics committee at Tabriz University of Medical Sciences and the approval number is TBZMED.REC.1394.1207. This study is done as a thesis to get the specialty degree in internal medicine.

\section{Statistical analysis}

Data gathered by the SPSS 18 program and analyzed by descriptive methods (mean \pm SD) and simple linear regression and multivariate stepwise linear regression analysis. A $P<0.05$ is regarded as significant.

\section{Results}

The characteristics of the study population before transplant and during 6 months follow up are presented in Table 1 . The causes of ESRD include diabetic nephropathy in 17 patients, hypertensive nephropathy in 6 patients, eclampsia in one patient and other causes such as collagen-vascular disease and glomerulonephritis in four patients. Around $23(82.1 \%)$ patients received phosphate supplements while 26 (92.85\%) patients received calcitriol to prevent hypophosphatemia after transplant.

FGF23 levels showed a marked decrease at the first week of follow up after renal transplantation, however, still had high levels and showed no statistically significant difference during 6-month follow up $(P=0.106)$. FGF23 levels before transplant showed a significant correlation with FGF23 levels in first week, third month and sixth month after transplant $(P<0.05)$. Serum 25 hydroxyvitamin $\mathrm{D}$ levels showed no significant difference before and during 6-month follow up after transplant. The difference between iPTH levels before and after transplant showed statistically a significant fall $(P=0.009)$ while 
Table 1. Clinical characteristics of patients before transplant and during 6-month follow up

\begin{tabular}{lcccc}
\hline & Before transplant & 1st week post-transplant & 3rd month post-transplant & 6th month post-transplant \\
\hline Age $(\mathrm{y})$ & $39.9 \pm 13.3(20-63)$ & & & \\
Duration on dialysis $(\mathrm{mon})$ & $22.39 \pm 19.9(0-84)$ & & & \\
FGF23 $(\mathrm{pg} / \mathrm{mL})$ & $1353.3 \pm 2892(19-10290)$ & $655.03 \pm 1718.81(15.75-6720)$ & $721.23 \pm 2333.16(18-11980)$ & $572.39 \pm 2265.55(10.5-12010)$ \\
$25 \mathrm{OH} \mathrm{D}(\mathrm{ng} / \mathrm{mL})$ & $17.74 \pm 9.38(6.7-41)$ & $14.63 \pm 6.39(6.6-31.8)$ & $14.94 \pm 6.22(7.4-28.5)$ & $17.78 \pm 9.58(6.1-43.7)$ \\
iPTH $(\mathrm{pg} / \mathrm{mL})$ & $161.82 \pm 248.24(3.1-1302)$ & $48.49 \pm 47.39(3.7-210.1)$ & $39.08 \pm 38.51(3.5-137.5)$ & $61.07 \pm 98.95(2.4-505.7)$ \\
Phosphorus $(\mathrm{mg} / \mathrm{dL})$ & $4.17 \pm 0.34(3.3-4.8)$ & $3.82 \pm 0.45(2.9-5.2)$ & $3.92 \pm 0.33(3.3-4.7)$ & $9.24 \pm 0.38(8.5-9.8)$ \\
\hline Calcium $(\mathrm{mg} / \mathrm{dL})$ & $9.25 \pm 0.4(8.6-10.1)$ & $9 \pm 0.42(8.5-9.7)$ & $9.26 \pm 0.39(8.5-10.1)$ \\
\hline
\end{tabular}

Data are represented as mean $\pm \mathrm{SD}$ (minimum-maximum).

mean iPTH levels were in normal range after transplant. Serum phosphorus levels showed statistically significant fall after transplantation even until sixth month of follow up $(P=0.006)$ despite its level was in normal range (because of use of phosphate supplement in $82.1 \%$ of patients). Calcium levels showed no significant difference before and after the transplant. None of the patients showed hypercalcemia. In multivariate analysis, levels of FGF23 before transplantation was related to lower phosphate levels at third month after transplant $(P<0.05)$. Additionally, FGF23 levels were high after transplantation. In addition, FGF23 levels had a correlation with lower phosphate levels. There was no statistically significant relationship between iPTH with phosphate levels before transplantation and during 6-month follow up after transplantation $(P>0.05)$. Moreover, no significant relationship between FGF23 levels with serum calcium and 25 hydroxyvitamin D before and after transplantation was detected. Duration of dialysis, age and gender were related neither to FGF23 levels nor phosphate levels before or after transplantation. Figure 1 shows the error bar charts for serum levels of FGF23, 25 hydroxyvitamin $\mathrm{D}, \mathrm{iPTH}$, calcium and phosphorous concentrations in all four measured periods.

\section{Discussion}

Kidney transplantation recovers many of the functions of the kidney thereby providing better life compared to ESRD. However, in the case of CKD-MBD, transplantation does not always seem to be an ideal ameliorating treatment. Hypophosphatemia is reported in about $90 \%$ of posttransplant patients (4). Persistent hyperparathyroidism has long been suggested to be the cause of hypophosphatemia. However, inappropriate urinary phosphate wasting has been reported to occur despite low levels of PTH (2). The role of FGF23 in CKD-MBD opened new aspects.
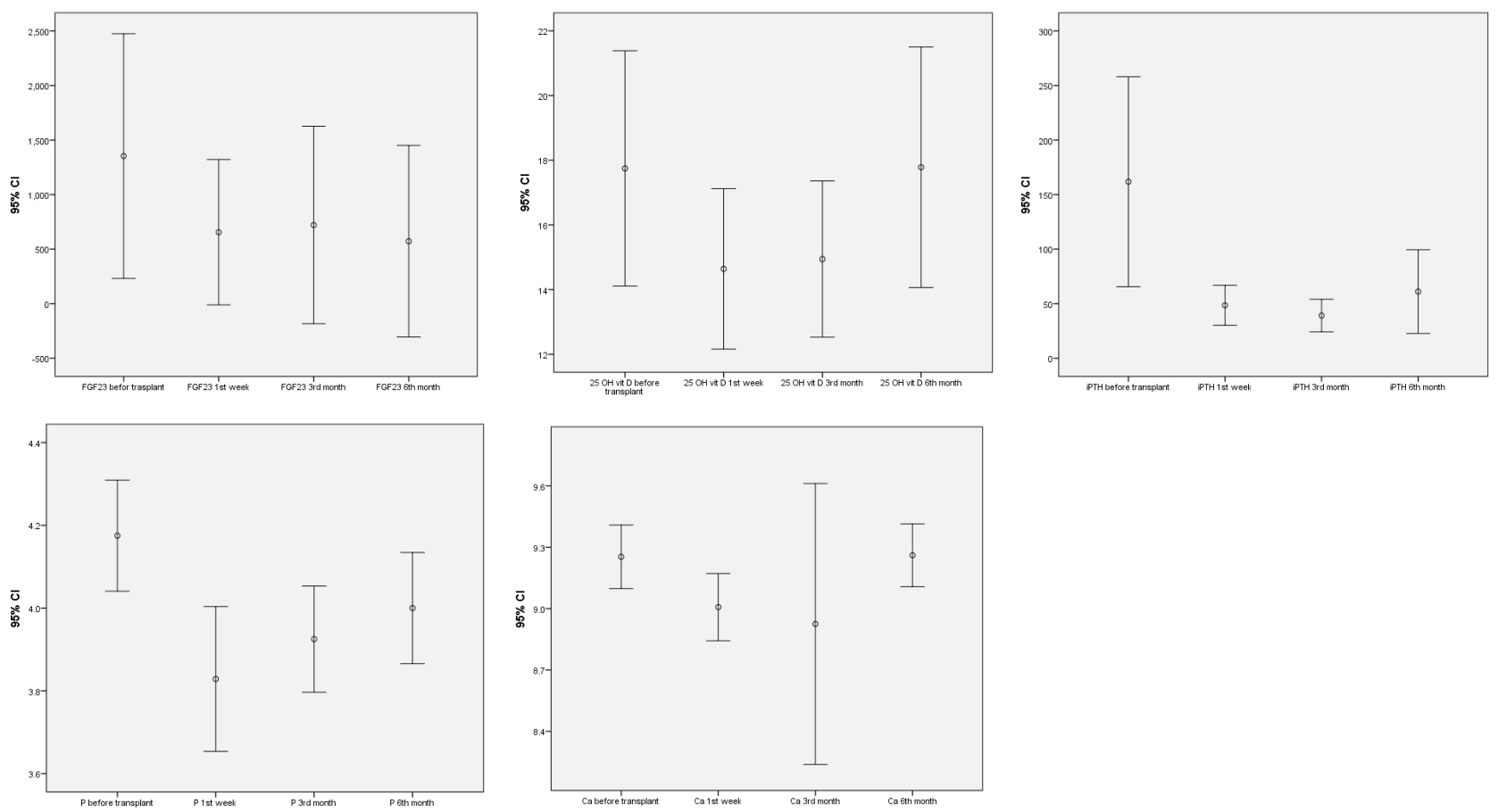

Figure 1. The error bar charts for levels of FGF23, 25OH vitD3, iPTH, calcium and phosphorous in all four times measured. 
In first study in the year 2006, Bhan et al (8) explained the $85 \%$ occurrence of hypophosphatemia after kidney transplantation and also a relationship between FGF23 levels before transplantation and presence of hypophosphatemia after renal transplantation. In the study by Evenepoel et al (6), a 95\% decrease in FGF23 levels after transplantation was detected, while its levels remained in ranges higher than normal in third month after transplantation. They found that levels of FGF23 before transplantation is the most important determinant factor for FGF23 concentration after transplantation. In our study, levels of FGF23 showed no significant difference after transplant and during 6 months of follow up. We also found a significant relationship between FGF23 levels before renal transplantation and its levels after renal transplantation. We concluded that high levels of FGF23 before transplantation in ESRD patients is an important determinant of high FGF23 levels after renal transplantation and in long-term follow up (6 months in this study). Besides, the strong relationship between FGF23 and phosphate levels before and after transplantation implies the fact that high levels of FGF23 in ESRD patients undergoing kidney transplantation predicts the occurrence of hypophosphatemia in long-term after kidney transplantation while high levels of FGF23 in any time after transplantation will cause hypophosphatemia. Like the previous studies, this study found no relationship between iPTH levels before transplantation and hypophosphatemia after renal transplantation. Other factors such as age, gender and duration of dialysis were not related to hypophosphatemia after kidney transplantation. Serum 25 hydroxyvitamin D and calcium levels showed also no significant difference before and after transplantation thus their role in hypophosphatemia were not determined in this study.

\section{Conclusion}

This study identified the role of high FGF23 levels in ESRD patients before transplantation in predicting persistent hypophosphatemia at three months after kidney transplant. Additionally we determined high levels of FGF23 in ESRD state as the most important predictor of high FGF23 levels during 6 months of follow up that cause persistent hypophosphatemia. These results are confined to this study. According to the limitations in the design of the study and the limitations we faced in interfering with a treatment plan and drugs administered to the patients. Thus larger studies with control group should be conducted to better clarify the impact of FGF23 after transplant.

\section{Limitations of the study}

Small number of subjects was a limitation for this study. Furthermore, this study was an observational one and we are not allowed to change the drug regimen or the supplements that were prescribed for most of the patients. Hence further studies on this subject are recommend.

Authors' contribution

SR and ZN designed the study, observed accuracy and validity of the study. $\mathrm{ZN}$ collected the data. A-GH performed the lab tests and MG analyzed the data. SR, JE, and SA supervised the project. SR and ZN wrote the paper. All authors edited and revised the final manuscript and accepted its publication.

\section{Conflicts of interest}

The authors declared no competing interests.

\section{Ethical considerations}

Ethical issues (including plagiarism, data fabrication, double publication) have been completely taken into account by the authors.

\section{Funding/Support}

This article is part of the thesis work of the internal medicine specialty of Zahra Navarbaf. It was funded with resources from Kidney Research Center of Tabriz University of Medical Sciences.

\section{References}

1. Nitta K, Nagano N, Tsuchiya K. Fibroblast growth factor 23/klotho axis in chronic kidney disease. Nephron Clin Pract. 2014;128:1-10. doi: 10.1159/000365787.

2. Seeherunvong W, Wolf M. Tertiary excess of fibroblast growth factor 23 and hypophosphatemia following kidney transplantation. Pediatr Transplant. 2011;15:37-46. doi: 10.1111/j.1399-3046.2010.01405.x.

3. Wesseling-Perry K, Pereira RC, Tsai E, Ettenger R, Jüppner H, Salusky IB. FGF23 and mineral metabolism in the early post-renal transplantation period. Pediatr Nephrol. 2013;28:2207-15. doi: 10.1007/s00467-013-2547-z.

4. Kawarazaki H, Shibagaki Y, Fukumoto S, Kido R, Nakajima I, Fuchinoue S, et al. The relative role of fibroblast growth factor 23 and parathyroid hormone in predicting future hypophosphatemia and hypercalcemia after living donor kidney transplantation: a 1-year prospective observational study. Nephrol Dial Transplant. 2011;26:2691-5. doi: $10.1093 /$ ndt/gfq777.

5. Rao M, Jain P, Ojo T, Surya G, Balakrishnan V. Fibroblast growth factor and mineral metabolism parameters among prevalent kidney transplant patients. Int J Nephrol. 2012; 2012:490623. doi: 10.1155/2012/490623.

6. Evenepoel P, Naesens M, Claes K, Kuypers D, Vanrenterghem Y. Tertiary 'hyperphosphatoninism' accentuates hypophosphatemia and suppresses calcitriol levels in renal transplant recipients. Am J Transplant. 2007;7:1193-200. doi: 10.1111/j.1600-6143.2007.01753.x.

7. Evenepoel P, Meijers BK, de Jonge H, Naesens M, Bammens B, Claes K, et al. Recovery of hyperphosphatoninism and renal phosphorus wasting one year after successful renal transplantation. Clin J Am Soc Nephrol. 2008;3:1829-36. doi: $10.2215 /$ CJN.01310308. 
8. Bhan I, Shah A, Holmes J, Isakova T, Gutierrez O, Burnett SA, et al. Post-transplant hypophosphatemia: tertiary 'hyper-phosphatoninism'? Kidney Int. 2006;70:1486-94. doi:10.1038/sj.ki.5001788.

9. Wolf M, Molnar MZ, Amaral AP, Czira ME, Rudas A, et al. Elevated fibroblast growth factor 23 is a risk factor for kidney transplant loss and mortality. J Am Soc Nephrol. 2011;22:956-66. doi: 10.1681/ASN.2010080894.

10. Bleskestad IH, Thorsen IS, Jonsson G, Skadberg Ø, Bergrem H, Gøransson LG. Soluble Klotho and intact fibroblast growth factor 23 in long-term kidney transplant patients. Eur J Endocrinol. 2015;172:343-50. doi: 10.1530/EJE-140457.

11. Prasad N, Jaiswal A, Agarwal V, Kumar S, Chaturvedi S, Yadav S, et al. FGF23 is associated with early posttransplant hypophosphataemia and normalizes faster than $\mathrm{iPTH}$ in living donor renal transplant recipients: a longitudinal follow-up study. Clin Kidney J. 2016; 9:669-76. doi: $10.1093 / \mathrm{ckj} / \mathrm{sfw} 065$.

12. Silver J, Naveh-Many T. FGF23 and the parathyroid. Adv Exp Med Biol. 2012;728:92-9. doi: 10.1007/978-1-46140887-1-6.

13. Lanske B, Razzaque MS. Molecular interactions of FGF23 and PTH in phosphate regulation. Kidney Int. 2014;86:1072-4. doi: 10.1038/ki.2014.316.

14. Rodríguez M, López I, Muñoz J, Aguilera-Tejero E, Almaden Y. FGF23 and mineral metabolism, implications in CKD-MBD. Nefrología (Madrid). 2012; 32:275-8. doi: 10.3265/Nefrologia.pre2012.Mar.11415.

15. Barros X, Torregrosa JV. Role of FGF23 in kidney transplantation. Transplant Technol Res. 2012;44:2551-4. doi: $\quad$ 10.1016/j.transproceed.2012.09.070.

16. Silver J, Rodriguez M, Slatopolsky E. FGF23 and PTHdouble agents at the heart of CKD. Nephrol Dial Transplant.
2012;27:1715-20. doi: 10.1093/ndt/gfs050.

17. Ibrahim S, Rashed L. Serum fibroblast growth factor-23 levels in chronic haemodialysis patients. Int Urol Nephrol. 2009;41:163-9. doi: 10.1007/s11255-008-9466-0.

18. Nakai K, Komaba H, Fukagawa M. New insights into the role of fibroblast growth factor 23 in chronic kidney disease. J Nephrol. 2010;23:619-25.

19. Isakova T. Fibroblast growth factor 23 and adverse clinical outcomes in chronic kidney disease. Curr Opin Nephrol Hypertens. 2012;21:334-40. doi: 10.1097/ MNH.0b013e328351a391.

20. Economidou D, Dovas S, Papagianni A, Pateinakis P, Memmos D. FGF-23 levels before and after renal transplantation. J Transplant. 2009;2009:379082. doi: $10.1155 / 2009 / 379082$.

21. Ketteler M, Petermann AT. Phosphate and FGF23 in early CKD: on how to tackle an invisible foe. Nephrol Dial Transplant. 2011;26:2430-2. doi: 10.1093/ndt/gfr344.

22. Lavi-Moshayoff V, Wasserman G, Meir T, Silver J, Naveh-Many T. PTH increases FGF23 gene expression and mediates the high-FGF23 levels of experimental kidney failure: a bone parathyroid feedback loop. Am J Physiol Renal Physiol. 2010;299:F882-9. doi: 10.1152/ ajprenal.00360.2010.

23. Wolf M. Update on fibroblast growth factor 23 in chronic kidney disease. Kidney Int. 2012;82:737-47. doi: 10.1038/ ki.2012.176.

24. Russo D, Battaglia Y. Clinical significance of FGF-23 in patients with CKD. Int J Nephrol. 2011;2011:364890. doi: 10.4061/2011/364890.

25. Fructuoso AI, Maestro ML, Pérez-Flores I, Valero R, Rafael S, Veganzones S, et al. Serum level of fibroblast growth factor 23 in maintenance renal transplant patients. Nephrol Dial Transplant. 2012;27:4227-35. doi: 10.1093/ndt/gfs409.

Copyright (C) 2019 The Author(s); Published by Nickan Research Institute. This is an open-access article distributed under the terms of the Creative Commons Attribution License (http://creativecommons.org/licenses/by/4.0), which permits unrestricted use, distribution, and reproduction in any medium, provided the original work is properly cited. 